\title{
Patrones espaciales de la riqueza específica de las culebras Thamnophis en México
}

\section{Spatial patterns of species richness of garter snakes Thamnophis in Mexico}

\author{
Crystian Sadiel Venegas-Barrera* y Javier Manjarrez \\ Facultad de Ciencias, Universidad Autónoma del Estado de México. Instituto Literario 100, Colonia Centro, Toluca 50000 Estado de México, México. \\ *Correspondencia: sadiel@juppa.unsij.edu.mx
}

\begin{abstract}
Resumen. Se identificaron tanto las áreas de mayor riqueza específica de las culebras del género Thamnophis y su presencia en las provincias biogeográficas y las Regiones Terrestres Prioritarias (RTP), como los factores antropogénicos que pueden influir en su distribución. Con la combinación de mapas de distribución potencial de las especies se creó un mapa de riqueza específica para calcular el porcentaje de celdas ocupadas por el género dentro de cada provincia biogeográfica, de cada RTP y conocer el tipo de uso de suelo. Además, se obtuvo la complementariedad de especies y la ordenación de las RTP a partir de su composición específica. El género se distribuyó en el 80.5\% de México, en todas sus provincias biogeográficas. Los estados de Durango, Michoacán, Puebla, Veracruz y Oaxaca presentaron la mayor riqueza específica. Potencialmente, el género puede encontrarse en el $96 \%$ de las RTP de México con un patrón altitudinal y climático. De 6 grupos de las RTP, el grupo oriental presentó la mayor riqueza específica. El $25.8 \%$ de la distribución potencial del género se encontró en áreas modificadas por el hombre. Se discuten los patrones de distribución bajo una perspectiva ecológica, biogeográfica y de conservación y se sugiere comprobar la riqueza específica potencial con estudios de campo.
\end{abstract}

Palabras clave: distribución espacial, regiones terrestres prioritarias, provincias biogeográficas, complementariedad, factores de riesgo.

\begin{abstract}
We identified areas of high species richness of snakes of the genus Thamnophis, their presence within biogeographic provinces and Terrestrial Priority Regions (TPR). We also identified anthropogenic factors that may influence their distribution. We created a map of species richness from the combination of potential distribution maps of species to calculate the percentage of cells occupied by the genus within each biogeographic province, TPR and type of land use. In addition, we obtained the complementarity of species and the ordination of TPR from its species composition. The genus is distributed in $80.5 \%$ of Mexico, in all biogeographic provinces. Durango, Michoacán, Puebla, Veracruz and Oaxaca had the highest species richness. Potentially, the genus can be found in $96 \%$ of the TPR of Mexico with a pattern altitude and climatic. From 6 groups of TPR, the Eastern group had the highest species richness. The $25.8 \%$ of the potential distribution of the genus is found in areas modified by man. We discuss the distribution patterns under an ecological, biogeographic and conservation approach and suggest checking the potential richness with field studies.
\end{abstract}

Key words: spatial distribution, priority terrestrial regions, biogeographic provinces, complementarity, risk factors.

\section{Introducción}

La historia geológica, la convergencia de biotas con distintas afinidades biogeográficas y la alta variación espacio-temporal de los recursos ambientales han favorecido que en México se presente un elevado número de especies de plantas y animales (CONABIO, 1998; Escalante et al., 2002; Álvarez-Mondragón y Morrone, 2004; Morrone, 2005; Ochoa-Ochoa y Flores-Villela, 2006). Sin embargo, existe una fuerte presión sobre los recursos ambientales como resultado de la actividad antropogénica que entre los años de 1976 y 2000 ha reducido un $20 \%$ de las selvas,

Recibido: 28 octubre 2009; aceptado: 22 abril 2010
$5 \%$ de los bosques y $8 \%$ de los matorrales (Torres, 2004). Adicionalmente, en el mismo periodo, los pastizales inducidos y las zonas de cultivo se incrementaron en un 33\% y $19 \%$, respectivamente, y se prevé una reducción de hasta el $90 \%$ de la distribución potencial del $2.4 \%$ de 334 especies endémicas de mamíferos, aves y mariposas, ante un escenario optimista de cambio climático (Peterson et al., 2002). Tal es el caso del noreste de México, donde la distribución real de aves y mamíferos posiblemente será menor que la potencial, como resultado de la modificación del medio (Ortega-Huerta y Peterson, 2004).

Ante el incremento en la degradación del medio es imperante ampliar el conocimiento de las áreas con mayor riqueza de especies (Peterson et al., 2002; Álvarez-Mon- 
dragón y Morrone, 2004; Ochoa-Ochoa y Flores-Villela, 2006). En México se reconoce el Eje Neovolcánico Transversal como centro de alta riqueza específica en distintos grupos taxonómicos (Badgley y Fox, 2000; Escalante et al., 2002; Álvarez-Mondragón y Morrone, 2004), como resultado de la coexistencia de los elementos paleoaméricano, neártico, mesoamericano tropical y mesoamericano de montaña (Morrone, 2005). Por ejemplo, la mayor riqueza de anfibios y reptiles de México se encuentra en esta región biogeográfica, pero también es donde se presenta la mayor densidad poblacional humana (Ochoa-Ochoa y Flores-Villela, 2006). Se tiene el convencimiento de que la mayor riqueza de especies de reptiles se encuentra en el Eje Neovolcánico Transversal (Ochoa-Ochoa y FloresVillela, 2006), pero aún es necesario identificar las áreas más ricas y las actividades de riesgo antropogénico para cada taxa de reptiles, debido a que cada grupo responde diferencialmente a los factores ambientales en función de sus requerimientos de nicho y por lo tanto las estrategias de conservación deben ser también específicas (Pulliam, 2000; Bestelmeyer et al., 2003).

Desde esta perspectiva es posible analizar el uso específico de los recursos espaciales de taxa relacionados filogenéticamente, identificando las regiones terrestres prioritarias, expresadas como RTP, y los estados de la República Mexicana con mayor riqueza, así como las actividades antropogénicas que pueden influir en su distribución geográfica, utilizando como modelo de estudio las especies de culebras del género Thamnophis presentes en México. Estas culebras son indicadoras de la calidad del medio acuático porque son de hábitos semiacuáticos y susceptibles a las modificaciones en el volumen, calidad y disponibilidad de los cuerpos de agua donde habitan (Rossman et al., 1996).

\section{Materiales y métodos}

Para el estudio, se digitalizaron los mapas de distribución potencial de las 25 especies del género Thamnophis (Rossman et al. 1996; Cuadro 1) registradas para México (Flores-Villela y Canseco-Márquez, 2004; Rossman y Burbrink, 2005), con una resolución espacial de 0.025 grados (celdas de aproximadamente $3 \times 3 \mathrm{~km}$ ). Se utilizaron las clasificaciónes taxonómicas propuestas por Rossman et al. (2005), Rossman y Brubrink (1996) y de Queiroz et al. (2002). Los mapas digitalizados fueron utilizados para calcular la frecuencia de la riqueza específica y la presencia de las especies en las provincias biogeográficas, en las Regiones Terrestres Prioritarias (RTP), en los estados de la República Mexicana, en áreas con agricultura (por humedal, riego y temporal), con pastizales (inducidos y cultivados), y en zonas urbanas. El mapa de riqueza específica se obtuvo de la suma de los 25 mapas de presencia/ ausencia, y se utilizó para calcular la frecuencia de celdas con $1,2,3, \ldots, n$ especies. El porcentaje de presencia de las especies por área analizada se estimó a partir del cociente del número de celdas en los que la especie estuvo y el número total de celdas del área analizada por 100 .

Para evidenciar la afinidad del género Thamnophis entre las provincias biogeográficas, se estimó el aporte de cada una a la distribución del género (sensu Arriaga et al., 2000) y se identificaron las provincias con la mayor riqueza específica. De igual forma, se evaluó el aporte de cada estado a la distribución del género en el país.

En el presente estudio se cuantifico el porcentaje de la distribución y la frecuencia de la riqueza específica contenida en las RTP, ya que estas áreas fueron decretadas para preservar la diversidad de especies (Arriaga et al., 2000) $\mathrm{y}$ por representar una mayor variedad de condiciones ambientales que las Áreas Naturales Protegidas (ANP). Estas últimas presentan una menor área superficial, representan una menor variedad de condiciones ambientales y algunas se decretaron con criterios distintos a la conservación de la diversidad (Ochoa-Ochoa y Flores-Villela, 2006).

Para identificar las RTP que aportan la mayor riqueza específica,se calculó el número de especies que hay en ellas y su contribución a la distribución del género, por estado. Para determinar que tan representadas se encuentran las especies dentro de las RTP se calculó el porcentaje de distribución de cada especie en las RTP. La ordenación de las RTP se realizó para agrupar a las que presentaran una composición de especies similar, lo que sugiere que poseen un nicho de requerimiento similar (Bestelmeyer et al., 2003). De tal forma, se aplicó el análisis de agrupamiento aglomerativo con la regla de amalgamación de Ward, que maximiza la varianza entre los grupos y la minimiza dentro de los grupos (Jongman et al. 1995), utilizando como medida de distancia el índice binario de Dice (presencia-ausencia de las especies de culebras, Jackson et al., 1989). La distancia de corte fue definida cuando la curva de acumulación de unión se estabilizó, la cual se utiliza para identificar el valor de distancia en la que la composición entre grupos es diferente.

La complementariedad se obtuvo por el método de Humphries et al. (1991), con el objetivo de identificar el menor número de RTP que contengan la mayor cantidad de especies en el área de estudio. Para ello se eligieron las 2 RTP que en conjunto presentaran el mayor número de especies; la primera fue la que presentó la mayor riqueza de especies y la segunda, la que contó con el mayor número de especies adicionales, es decir aquellas que no están 
Cuadro 1. Porcentaje de presencia de las especies del género Thamnophis en México, en las Regiones Terrestres Prioritarias (RTP) y por tipo de uso de suelo

\begin{tabular}{|c|c|c|c|c|c|}
\hline \multirow[t]{2}{*}{ Especie } & \multirow[t]{2}{*}{ México $^{a}$} & \multirow[t]{2}{*}{$R T P^{b}$} & \multicolumn{3}{|c|}{ Tipo de Uso de Suelo } \\
\hline & & & Agrícola & Pastizal & Urbano \\
\hline T. bogerti* & 0.98 & 48.2 & 38.1 & 6.1 & 0.1 \\
\hline T. chrysocephalus ${ }^{* 1}$ & 3.42 & 40.3 & 27.0 & 6.6 & 0.1 \\
\hline T. conanti ${ }^{*}$ & 0.20 & 36.4 & 30.0 & 2.3 & 0.7 \\
\hline T. cyrtopsis $^{l}$ & $\mathbf{5 4 . 0 0}$ & 25.3 & 19.6 & 4.7 & 0.3 \\
\hline T. elegans ${ }^{1}$ & 0.16 & 45.4 & 0.9 & 0.0 & 0.0 \\
\hline T. eques ${ }^{1}$ & 28.81 & 21.9 & 23.9 & 3.8 & 0.5 \\
\hline T. errans $* 2$ & 4.24 & 41.2 & 4.9 & 1.6 & 0.0 \\
\hline T. exsul*2 & 0.02 & 50.0 & 3.1 & 3.1 & 0.0 \\
\hline T. fulvus ${ }^{1}$ & 1.52 & 5.5 & 6.9 & 0.7 & 0.1 \\
\hline T. godmani* & 0.28 & 0.1 & 9.0 & 1.4 & 0.3 \\
\hline T. hammondii ${ }^{l}$ & 1.34 & 28.3 & 10.0 & 3.1 & 0.9 \\
\hline T. lineri $^{*}$ & 0.14 & 85.9 & 17.0 & 4.0 & 0.0 \\
\hline T. marcianus $^{I}$ & 31.47 & 21.2 & 10.7 & 6.0 & 0.3 \\
\hline T. melanogaster ${ }^{* 2}$ & 12.27 & 10.8 & 34.6 & 5.4 & 0.8 \\
\hline T. mendax $^{* 2}$ & 0.04 & 94.0 & 12.8 & 0.0 & 0.0 \\
\hline T. nigronuchalis ${ }^{* 2}$ & 0.51 & 10.8 & 1.5 & 3.6 & 0.0 \\
\hline T. postremus* & 0.18 & 6.1 & 39.4 & 5.5 & 0.0 \\
\hline T. proximus ${ }^{3}$ & 16.01 & 27.2 & 18.0 & 24.3 & 0.3 \\
\hline T. pulchrilatus ${ }^{* I}$ & 1.71 & 34.0 & 35.8 & 7.3 & 0.5 \\
\hline T. rufipunctatus ${ }^{* 2}$ & 10.67 & 21.2 & 10.0 & 0.9 & 0.1 \\
\hline T. scalaris $^{* 2}$ & 2.54 & 21.2 & 49.5 & 8.1 & 2.1 \\
\hline T. scaliger ${ }^{* 2}$ & 0.62 & 10.7 & 48.3 & 10.7 & 9.3 \\
\hline T. sirtalis ${ }^{3}$ & 0.28 & 70.5 & 7.5 & 0.0 & 0.3 \\
\hline T. sumichrasti ${ }^{* 2}$ & 0.96 & 36.6 & 38.0 & 10.1 & 0.5 \\
\hline T. validus ${ }^{* 2}$ & 3.87 & 19.7 & 35.0 & 3.1 & 0.5 \\
\hline Género & 80.4 & 70.8 & 17.8 & 7.6 & 0.3 \\
\hline
\end{tabular}

a porcentaje de la distribución de las especies en México, ${ }^{\mathrm{b}}$ porcentaje de la distribución de las especies en las RTP, ${ }^{\mathrm{c}}$ porcentaje de la distribución de las especies por tipo de uso de suelo,

* endémica de México, ${ }^{1}$ clado de amplia distribución, ${ }^{2}$ clado mexicano $\mathrm{y}^{3}$ grupo hermano del resto de las especies del género Thamnophis (de Queroiz, et al. 2002). En negritas,los valores más altos. 
representadas en la primera RTP. Al número de especies no compartidas entre las 2 RTP, se le denominó número de especies complementarias. Para establecer la prioridad entre las RTP se empleó el complemento residual, que es el valor de la diferencia entre el número total de especies disponibles y el número de especies complementarias (Álvarez-Mondragón y Morrone, 2004). La elección del número mínimo de RTP que contuvieran todas las especies se obtuvo a partir de la porción complementaria acumulada, la que a su vez se obtuvo a partir de la suma de las especies complementarias de las RTP elegidas. El proceso de elección consistió en elegir la RTP que presentará el valor de complemento residual menor, luego se eligió la que contará con el segundo número máximo de especies complementarias, es decir, aquella que contará con el mayor número de especies no compartidas. El mismo criterio fue utilizado iterativamente hasta elegir el número menor de RTP que contuvieran las 25 especies de culebras del género Thamnophis.

Se utilizó la presencia de las zonas agrícolas, urbanas y pastizales inducidos-cultivados como indicadores indirectos de la presión antropogénica sobre los cuerpos de agua. Con el objetivo de estimar el nivel de impacto antropogénico en las RTP se obtuvo el número de poblados, el número de habitantes y la densidad poblacional en las RTP, utilizando el mapa de CONABIO de los poblados de México para el año 2000. Adicionalmente, se estimó el porcentaje de convergencia entre la distribución de cada una de las especies y del género con áreas agrícolas (por humedal, riego y temporal), pastizales (inducidos y cultivados) y zonas urbanas. Los mapas de las entidades federativas, RTP, provincias biogeográficas y uso de suelo fueron obtenidos de la mapoteca digital de CONABIO (www.conabio.gob.mx).

\section{Resultados}

El género Thamnophis se distribuyó en el $80.5 \%$ de la República Mexicana (Fig. 1a y Cuadro 1) y presentó el mayor porcentaje de celdas con más de 4 especies (Fig. 1b). Thamnophis cyrtopsis, T. marcianus y T. eques fueron las especies con la mayor distribución geográfica en el país, mientras que las de menor distribución fueron $T$. exsul, T. mendax y T. elegans (Cuadro 1). El género estuvo presente en todas las provincias biogeográficas de México, ocupando menos del 33\% del área en las del Cabo, Yucatán, California y Petén (Fig. 2). En contraste, el género puede estar presente en más del 95\% del área de las provincias Tamaulipeca, Sierra Madre del Sur, Depresión del Balsas, Altiplano del Norte, Oaxaca, el Eje Neovolcánico
Transversal, y las Sierras Madre Oriental y Occidental, donde se presentó el mayor número de especies (Fig. 2). La Sierra Madre Occidental fue la provincia que aportó el mayor porcentaje de distribución del género en México (13.7\%), mientras que el menor lo aportó la de Baja California $(0.7 \%$, Fig. 2$)$.

Los estados de Chihuahua, Sonora y Coahuila aportaron el mayor número de celdas a la distribución del género, mientras que los de Aguascalientes, Colima, Morelos y Yucatán aportaron el menor (Cuadro 2). La mayor riqueza de especies se registró en Durango, Michoacán, Puebla, Veracruz y Oaxaca (Cuadro 1); en contraste, en los estados de Baja California Sur, Campeche, Quintana Roo, Tabasco y Yucatán se registró el menor número de especies (Fig. 1).

Al analizar la distribución del género en las RTP, se encontró que el género potencialmente puede estar en el $96 \%$ de las RTP de México y en cerca del $70.8 \%$ de sus celdas (Fig. 3a y Cuadro 1). En México, más del 50\% de la distribución geográfica de Thamnophis lineri, T. mendax, T. elegans y T. exsul se encontró en las RTP; mientras que menos del $10 \%$ de la distribución de las especies $T$. godmani, T. fulvus y T. postremus coincidió con las RTP (Cuadro 1), Las áreas ocupadas por el género, parecen no seguir un patrón latitudinal, pero sí uno altitudinal, debido a que la mayor riqueza específica se encontró en las RTP con altitudes mayores de $1000 \mathrm{~m}$ nsm. Por ejemplo, la mayor riqueza específica en las RTP se concentró en los estados de Veracruz, Puebla y Estado de México, los cuales presentan elevaciones máximas entre los 3000 y los $4000 \mathrm{~m}$ snm y potencialmente pueden encontrarse entre 6 y 7 especies por celda (Cuadro 2). En contraste, el menor número de especies se registró en las RTP de los estados con bajas elevaciones, como Baja California Sur, Campeche, Sinaloa, Tabasco y Yucatán.

Se formaron 6 grupos de RTP a partir del análisis de agrupamiento de la composición de especies de culebras del género Thamnophis (Fig. 3a), el valor de corte fue a las 5 unidades de la distancia de unión (Fig. 3b). Los grupos de RTP, que se describen a continuación, presentaron una tendencia espacial definida (Fig. 3a): a) grupo norteño, compuesto por 18 RTP que se localizan en los estados de Sonora, Chihuahua y Coahuila, en las cuales se encontraron 2 especies de amplia distribución; b) grupo atlántico, constituido por 20 RTP del las costas del golfo de México y sureste mexicano, en las cuales se encontraron 2 especies de amplia distribución; c) grupo oriental, con 30 RTP localizadas principalmente en la Sierra Madre Oriental y las Sierras de Oaxaca, donde potencialmente se pueden encontrar 16 especies; $d$ ) grupo californiano, con 11 RTP en la península de Baja California, excepto la región del Cabo, donde se pueden encontrar 3 especies de culebras; e) grupo pacífico, 29 RTP que se encuentran cercanas a 
Cuadro 2. Aporte en porcentaje del área de las RTP a la distribución estatal de las especies del género Thamnophis, por número de especies, número de poblados (NdP), miles de habitantes $(\mathrm{H})$, habitantes por celda (D) en las RTP, así como número de especies (NE), porcentaje que aporta el estado a la distribución del género en México (A) y porcentaje del estado ocupado por el género (O)

\begin{tabular}{|c|c|c|c|c|c|c|c|c|c|c|c|}
\hline \multirow{3}{*}{ Nombre del estado } & \multicolumn{8}{|c|}{$R T P$} & \multicolumn{3}{|c|}{ Estado } \\
\hline & \multicolumn{5}{|c|}{ Número de especies } & \multicolumn{3}{|c|}{ Actividad antropogénica } & \multirow[t]{2}{*}{$N E$} & \multirow[t]{2}{*}{$A$} & \multirow[t]{2}{*}{$O$} \\
\hline & 1 & 2 & 3 & 4 & $>5$ & $N d P$ & $H$ & $D$ & & & \\
\hline Aguascalientes & 0.0 & 0.0 & 17.7 & 1.3 & 0.0 & 27 & 1 & 0.1 & 4 & 0.3 & 99 \\
\hline $\mathrm{BC}$ & 9.3 & 1.1 & 0.0 & 0.0 & 0.0 & 309 & 14 & 0.1 & 3 & 2.0 & 41 \\
\hline $\mathrm{BCS}$ & 1.4 & 0.0 & 0.0 & 0.0 & 0.0 & 448 & 48 & 0.5 & 2 & 0.2 & 4 \\
\hline Campeche & 4.1 & 0.0 & 0.0 & 0.0 & 0.0 & 778 & 78 & 1.1 & 1 & 0.4 & 13 \\
\hline Chiapas & 6.7 & 1.1 & 0.3 & 0.0 & 0.0 & 5532 & 842 & 9.4 & 4 & 1.4 & 32 \\
\hline Chihuahua & 4.3 & 4.7 & 5.4 & 9.1 & 1.9 & 3542 & 167 & 0.5 & 6 & 16.3 & 100 \\
\hline Coahuila & 9.3 & 16.4 & 5.4 & 0.0 & 0.0 & 1448 & 248 & 1.3 & 7 & 9.6 & 98 \\
\hline Colima & 2.1 & 6.4 & 0.0 & 0.0 & 0.0 & 76 & 13 & 1.9 & 3 & 0.3 & 96 \\
\hline $\mathrm{DF}$ & 0.0 & 0.0 & 1.0 & 16.7 & 8.4 & 48 & 1 & 0.4 & 6 & 0.1 & 100 \\
\hline Durango & 1.9 & 2.8 & 10.5 & 2.7 & 0.7 & 1305 & 110 & 0.7 & 9 & 7.8 & 99 \\
\hline Guanajuato & 0.0 & 0.4 & 6.1 & 0.4 & 0.0 & 319 & 110 & 2.9 & 5 & 1.9 & 99 \\
\hline Guerrero & 15.8 & 7.3 & 0.7 & 0.0 & 0.0 & 1653 & 312 & 4.0 & 7 & 3.8 & 99 \\
\hline Hidalgo & 1.8 & 13.4 & 5.9 & 1.3 & 0.0 & 1169 & 263 & 10.1 & 7 & 1.2 & 95 \\
\hline Jalisco & 8.7 & 4.3 & 4.1 & 0.0 & 0.0 & 1303 & 368 & 3.7 & 7 & 4.8 & 98 \\
\hline México & 5.3 & 2.4 & 3.0 & 5.2 & 7.0 & 719 & 434 & 15.7 & 6 & 1.2 & 90 \\
\hline Michoacán & 10.6 & 2.2 & 3.9 & 1.0 & 0.3 & 1827 & 396 & 5.4 & 8 & 3.5 & 97 \\
\hline Morelos & 8.7 & 5.3 & 2.0 & 11.1 & 0.0 & 183 & 62 & 10.4 & 5 & 0.3 & 100 \\
\hline Nayarit & 1.6 & 14.5 & 7.3 & 0.5 & 0.0 & 582 & 91 & 2.6 & 6 & 1.7 & 97 \\
\hline Nuevo León & 2.9 & 6.5 & 5.0 & 0.0 & 0.0 & 663 & 87 & 1.0 & 5 & 3.7 & 90 \\
\hline Oaxaca & 15.0 & 14.9 & 12.7 & 0.0 & 0.0 & 3683 & 782 & 6.9 & 7 & 4.8 & 86 \\
\hline Puebla & 1.5 & 9.1 & 5.4 & 3.4 & 2.0 & 1435 & 602 & 14.3 & 9 & 2.1 & 100 \\
\hline Querétaro & 0.0 & 27.9 & 12.0 & 0.0 & 0.0 & 833 & 317 & 21.9 & 6 & 0.7 & 100 \\
\hline Quintana Roo & 26.9 & 4.8 & 0.0 & 0.0 & 0.0 & 964 & 275 & 5.1 & 2 & 1.4 & 53 \\
\hline SLP & 3.0 & 5.7 & 4.7 & 0.0 & 0.0 & 1242 & 307 & 4.0 & 6 & 3.3 & 86 \\
\hline Sinaloa & 5.5 & 9.3 & 0.0 & 0.0 & 0.0 & 636 & 106 & 1.5 & 5 & 3.5 & 97 \\
\hline Sonora & 5.5 & 7.1 & 5.5 & 0.2 & 0.0 & 638 & 86 & 0.4 & 7 & 9.4 & 78 \\
\hline Tabasco & 18.5 & 0.3 & 0.0 & 0.0 & 0.0 & 475 & 181 & 6.0 & 2 & 1.2 & 80 \\
\hline Tamaulipas & 0.3 & 16.4 & 1.4 & 0.8 & 0.5 & 949 & 95 & 1.0 & 6 & 4.8 & 97 \\
\hline Tlaxcala & 0.0 & 0.0 & 8.6 & 1.6 & 0.0 & 38 & 4 & 0.8 & 5 & 0.2 & 100 \\
\hline Veracruz & 7.8 & 0.3 & 0.8 & 0.4 & 1.7 & 1839 & 537 & 6.1 & 8 & 3.9 & 89 \\
\hline Yucatan & 2.2 & 0.0 & 0.0 & 0.0 & 0.0 & 56 & 62 & 1.3 & 2 & 0.6 & 24 \\
\hline Zacatecas & 0.0 & 0.2 & 1.9 & 0.7 & 0.0 & 113 & 24 & 0.3 & 6 & 3.1 & 66 \\
\hline
\end{tabular}

En negritas, los valores más altos. 


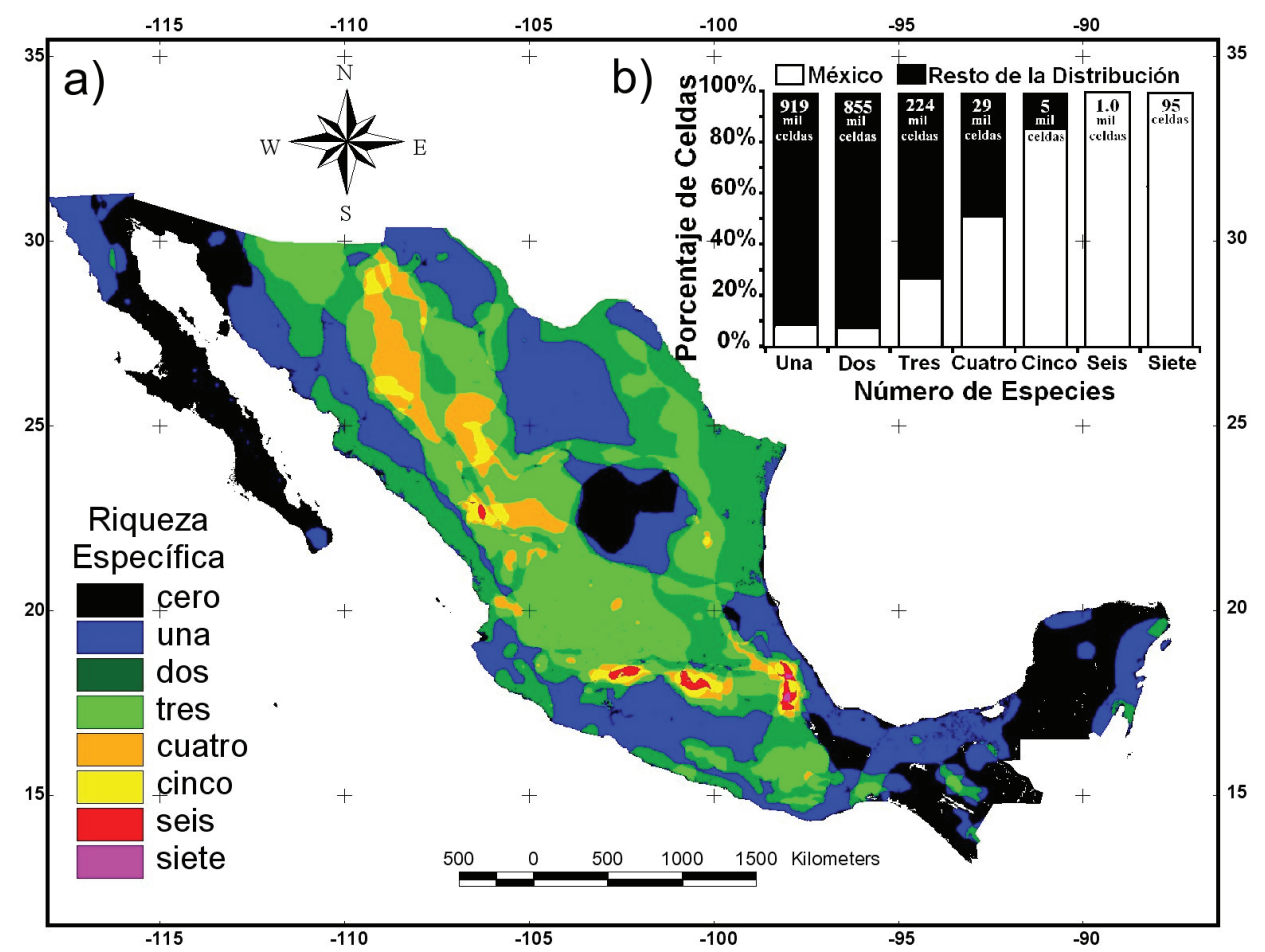

Figura 1. Mapa de la riqueza específica de las especies del género Thamnophis en México (a) y su aporte a la distribución del género en Norte y Centro América por número de especies (b).

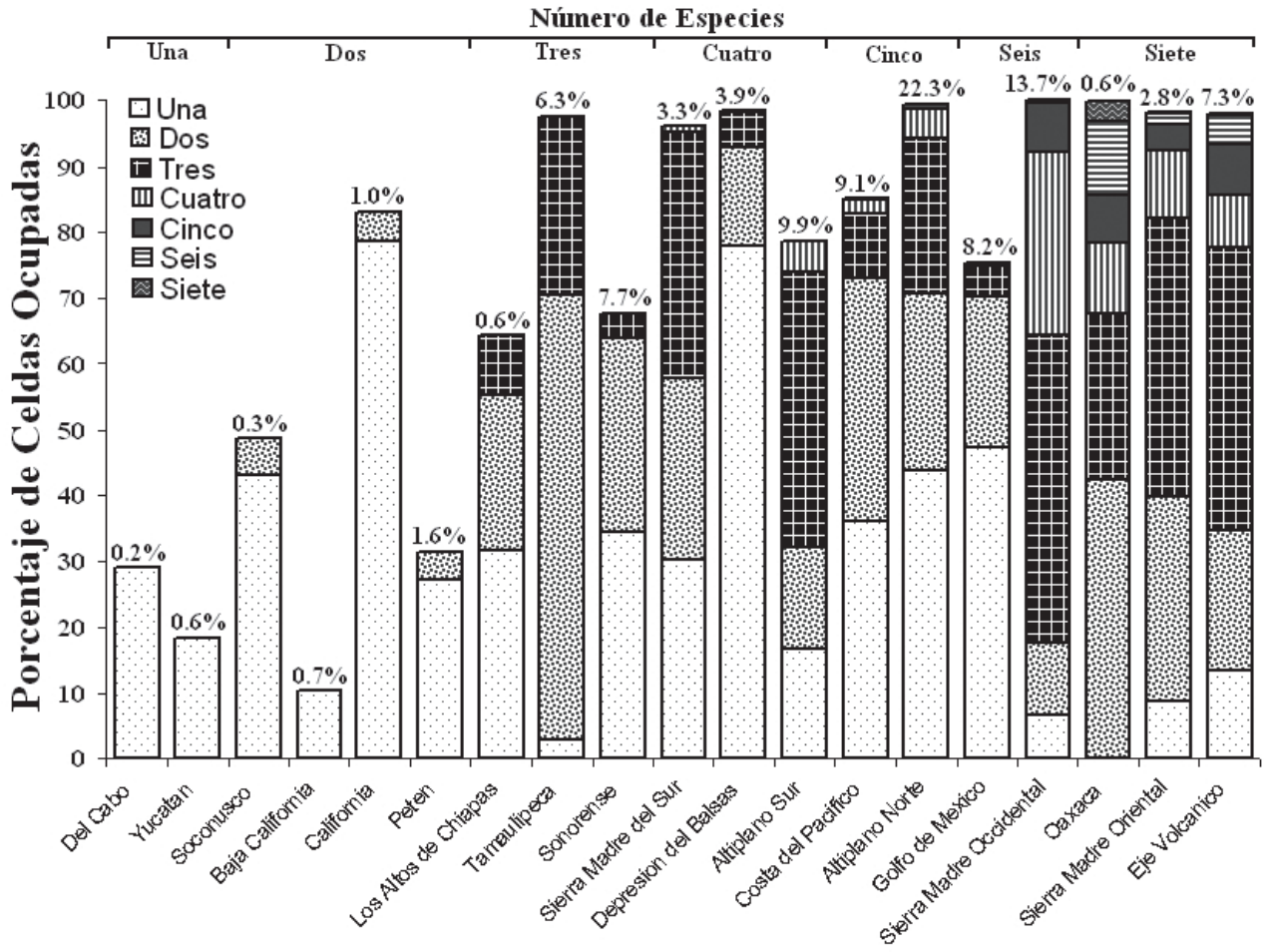

Provincia Biogeográfica

Figura 2. Porcentaje de celdas ocupadas por número de especies en las provincias biogeográficas. El valor sobre la barra indica el porcentaje de celdas que aporta la provincia a la distribución del género a México. 
Cuadro 3. Porcentaje y frecuencia de las especies del género Thamnophis en los grupos de RTP (obtenidos del análisis de agrupamiento aglomerativo)

\begin{tabular}{|c|c|c|c|c|c|c|c|}
\hline \multirow{2}{*}{ Especie } & \multicolumn{7}{|c|}{ Grupo de Región Terrestre Prioritaria } \\
\hline & $N$ & $A$ & $O$ & $C$ & $P$ & $\mathrm{CO}$ & Frecuencia \\
\hline T. bogerti & 0.0 & 0.0 & $\mathbf{5 0 . 0}$ & 0.0 & 50.0 & 0.0 & 2 \\
\hline T. chrysocephalus & 0.0 & 0.0 & 36.4 & 0.0 & 63.6 & 0.0 & 11 \\
\hline T. conanti & 0.0 & 0.0 & 20.0 & 0.0 & 80.0 & 0.0 & 5 \\
\hline T. cyrtopsis & 11.0 & 0.0 & 27.0 & 0.0 & 25.0 & 37.0 & 100 \\
\hline T. elegans & 0.0 & 0.0 & 0.0 & 100.0 & 0.0 & 0.0 & 1 \\
\hline T. eques & 0.0 & 0.0 & 26.9 & 0.0 & 1.9 & 71.2 & 52 \\
\hline T. errans & 0.0 & 0.0 & 0.0 & 0.0 & 5.6 & 94.4 & 18 \\
\hline T. exsul & 0.0 & 0.0 & 100.0 & 0.0 & 0.0 & 0.0 & 1 \\
\hline T. fulvus & 0.0 & 0.0 & 0.0 & 0.0 & 100.0 & 0.0 & 8 \\
\hline T. godmani & 0.0 & 0.0 & 0.0 & 0.0 & 100.0 & 0.0 & 1 \\
\hline T. hammondii & 0.0 & 0.0 & 0.0 & 100.0 & 0.0 & 0.0 & 11 \\
\hline T. lineri & 0.0 & 0.0 & 100.0 & 0.0 & 0.0 & 0.0 & 2 \\
\hline T. marcianus & 30.0 & 21.7 & 35.0 & 1.7 & 1.7 & 10.0 & 60 \\
\hline T. melanogaster & 0.0 & 0.0 & 12.5 & 0.0 & 4.2 & 83.3 & 24 \\
\hline T. mendax & 0.0 & 0.0 & 100.0 & 0.0 & 0.0 & 0.0 & 2 \\
\hline T. nigronuchalis & 0.0 & 0.0 & 0.0 & 0.0 & 0.0 & 100.0 & 2 \\
\hline T. postremus & 0.0 & 0.0 & 0.0 & 0.0 & 50.0 & $\mathbf{5 0 . 0}$ & 2 \\
\hline T. proximus & 0.0 & 39.6 & 54.2 & 0.0 & 6.3 & 0.0 & 48 \\
\hline T. pulchrilatus & 0.0 & 0.0 & 35.0 & 0.0 & 0.0 & 65.0 & 20 \\
\hline T. rufipunctatus & 0.0 & 0.0 & 5.6 & 0.0 & 0.0 & 94.4 & 18 \\
\hline T. scalaris & 0.0 & 0.0 & 30.0 & 0.0 & 10.0 & 60.0 & 10 \\
\hline T. scaliger & 0.0 & 0.0 & 0.0 & 0.0 & 0.0 & 100.0 & 3 \\
\hline T. sirtalis & 0.0 & 0.0 & 0.0 & 0.0 & 0.0 & 100.0 & 3 \\
\hline T. sumichrasti & 0.0 & 0.0 & 100.0 & 0.0 & 0.0 & 0.0 & 6 \\
\hline T. validus & 0.0 & 0.0 & 8.3 & 0.0 & 75.0 & 16.7 & 12 \\
\hline Número de especies & 2 & 2 & 16 & 3 & 14 & 13 & \\
\hline RTP & 18 & 20 & 30 & 11 & 29 & 38 & \\
\hline
\end{tabular}

N, norteño; A, atlántico; O, Oriental; C, Californiano; P, Pacífico; CO, Central-occidental. En negritas, los valores más altos. 
las costas del Pacifico y en las Sierras de Oaxaca, en las cuales se pueden encontrar 14 especies; f) grupo centraloccidental, compuesto por 38 RTP que se localizan en la Sierra Madre Occidental y el Eje Neovolcánico Transversal, donde se pueden encontrar 13 especies (Cuadro 3, Fig. 3a). Thamnophis marcianus fue la especie presente en los 6 grupos de RTP, mientras que 11 especies de culebras sólo estuvieron en uno de los grupos (Cuadro 3). El grupo oriental presentó la mayor riqueza de especies en comparación con los otros $5\left(X_{\text {g.l. }}=5=35.9, P<0.001\right)$.

La menor riqueza de especies de culebras (entre 1 y 2 especies) en las RPT fue la de los grupos norteño, atlántico, californiano y pacífico. Por el contrario, la mayor riqueza de especies se encontró en los grupos oriental, pacífico y central-occidental, en los cuales se pueden encontrar potencialmente hasta 7 especies por celda (Fig. 4). Finalmente, fueron necesarias 11 RTP para representar todas las especies del género Thamnophis, siendo las de Sierras del Norte de Oaxaca-Mixe, el Pico de Orizaba y Bavispe, las que aportaron el mayor número de especies complementarias y especies totales, así como el menor valor de complementos residuales (Cuadro 4).

En relación con la actividad antropogénica, el género está presente en cerca del $87.2 \%$ de las áreas modificadas para la agricultura, pastizales o en áreas urbanas de
México. En las RTP de los estados del centro del país, Hidalgo, Estado de México, Puebla y Querétaro, se presentó la mayor densidad poblacional (Cuadro 2), mientras que el mayor número de poblados fue registrado en las de los estados fronterizos de Chiapas y Chihuahua.

El $25.8 \%$ de la distribución potencial del género se encontró en áreas modificadas por el hombre, ya sea por la agricultura $(17.8 \%)$, áreas urbanas $(0.3 \%)$ o pastizales inducidos o cultivados (7.6\%, Cuadro 1). El 40\% de la distribución geográfica de Thamnophis scalaris y T. scaliger coincidió con áreas modificadas para la agricultura, más del $5 \%$ con pastizales inducidos o cultivados y más del 2\% con áreas urbanas. En contraste, la distribución de T. godmani, T. exsul y $T$. nigronuchalis presentó una baja coincidencia con áreas modificadas por el hombre (Cuadro 1). En los 3 tipos de uso de suelo se pueden encontrar hasta 7 especies y en cerca del $79.7 \%$ de estas áreas se pueden encontrar de 1 a 3 especies.

\section{Discusión}

El identificar las áreas de mayor riqueza específica es una primera aproximación para tener un mejor cono-

Cuadro 4. Complementariedad, complementos residuales y riqueza específica de las Regiones Terrestres Prioritarias (RTP) que contienen las 25 especies de culebras del género Thamnophis

\begin{tabular}{lcccc}
\hline \multicolumn{1}{c}{ RTP } & Grupo de RTP & $\begin{array}{c}\text { Número de especies } \\
\text { complementarias }\end{array}$ & $\begin{array}{c}\text { Complementos } \\
\text { residuales }\end{array}$ & Riqueza especíica \\
\hline Sierras del Norte Oaxaca-Mixe & Oriental & 8 & 17 & 8 \\
Pico de Orizaba-Cofre de Perote & Oriental & 3 & 22 & 8 \\
Bavispe-El Tigre & Central-occidental & 3 & 22 & 6 \\
Sierra de Chincua & Central-occidental & 2 & 23 & 6 \\
Río Presidio & Central-occidental & 2 & 23 & 6 \\
El Cielo & Oriental & 2 & 23 & 3 \\
Altos de Chiapas & Pacífico & 1 & 24 & 3 \\
Cañón del Zopilote & Pacífico & 1 & 24 & 2 \\
Sierra de Juárez & Californiano & 1 & 24 & 2 \\
Sierra de San Pedro Mártir & Californiano & 1 & 24 & 2 \\
Infiernillo & Pacífico & 1 & 24 & 6 \\
\hline
\end{tabular}




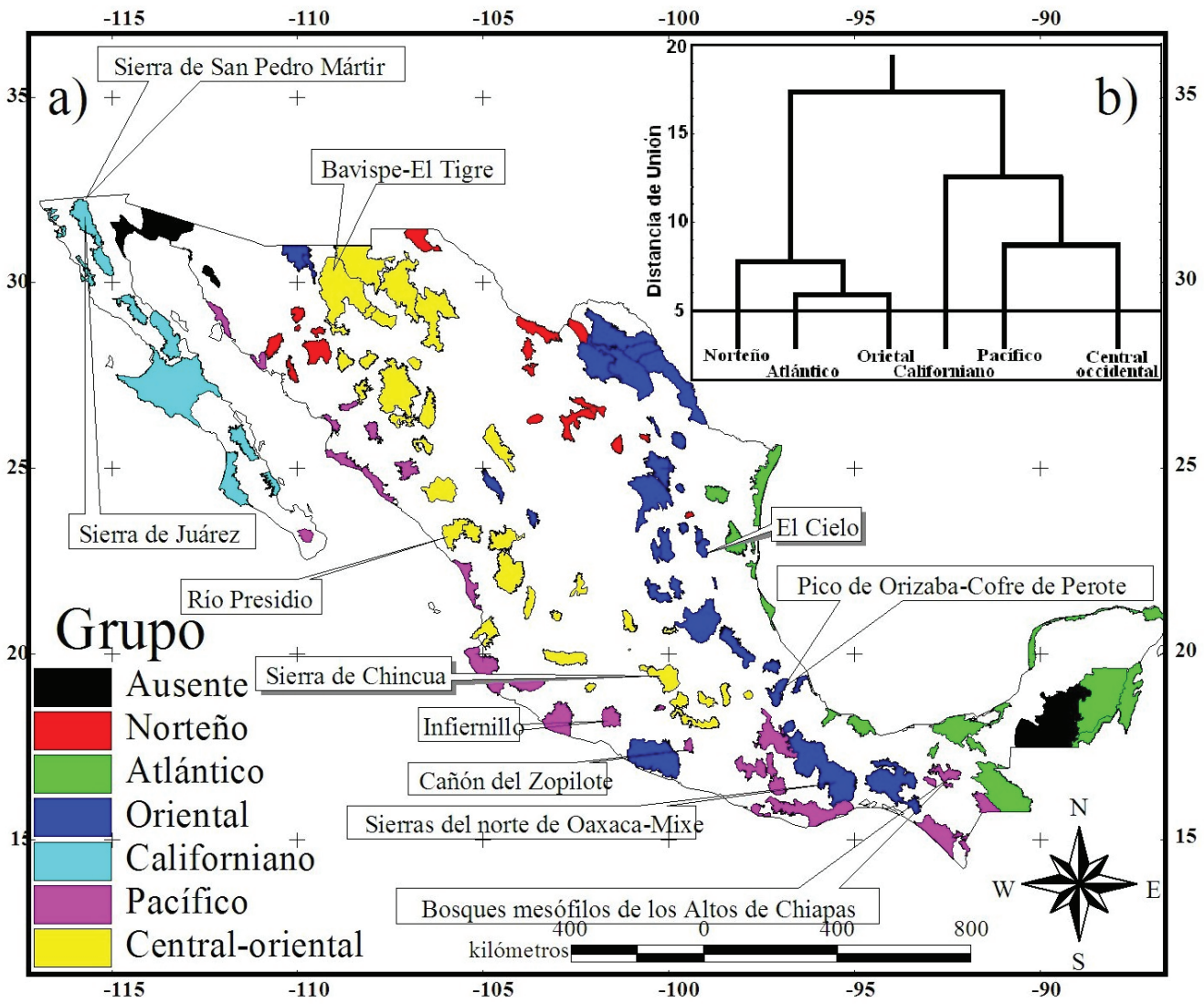

Figura 3. Localización de los grupos de Regiones Terrestres Prioritarias (RTP) en México; (a), se muestran las que aportan la mayor complementariedad de especies y (b), gráfica de agrupamiento de las RTP.

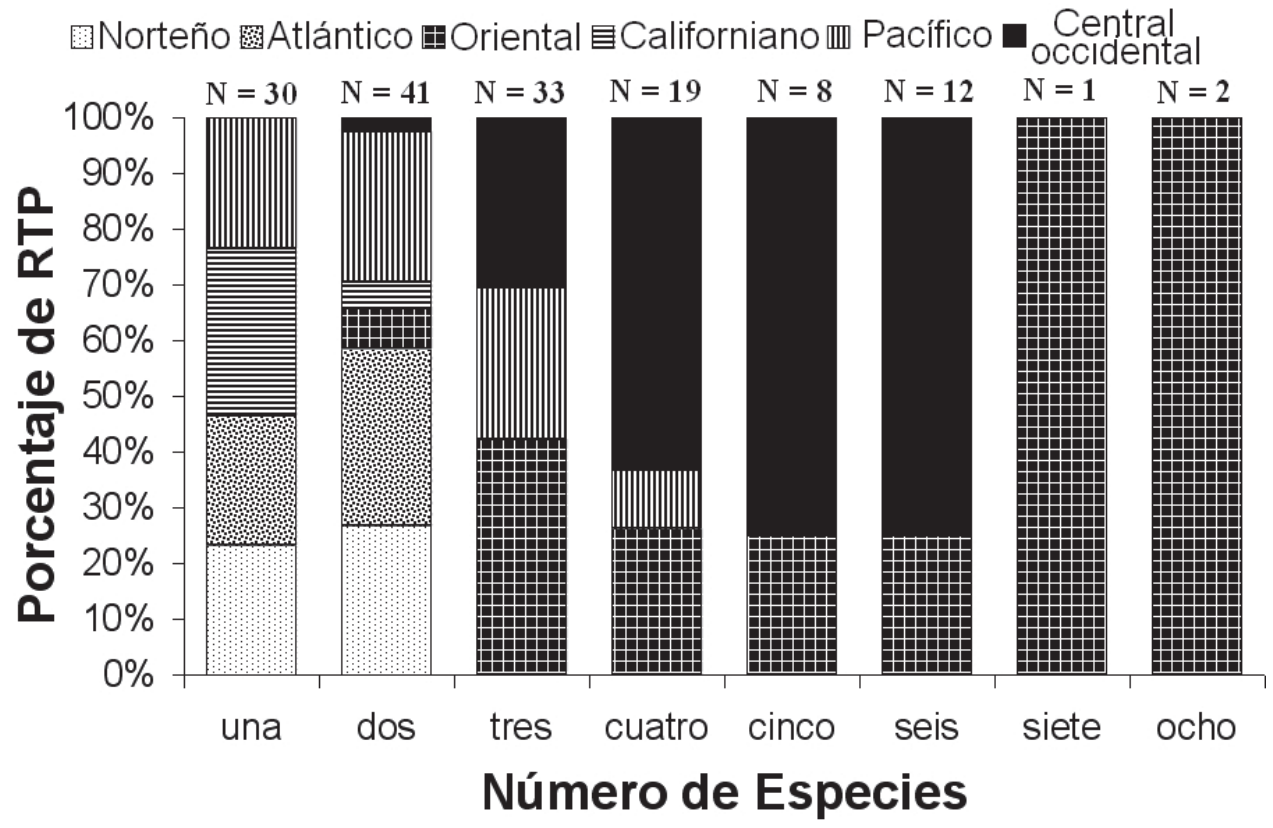

Figura 4. Porcentaje de grupos de Regiones Terrestres Prioritarias (RTP) por número de especies. 
cimiento de los patrones y mecanismos que determinan la distribución de las especies (Escalante et al., 2002; Álvarez-Mondragón y Morrone, 2004; Morrone, 2005; Ochoa-Ochoa y Flores-Villela, 2006). La tendencia actual en la conservación es analizar el mismo fenómeno biológico bajo distintos enfoques, con el objetivo de fortalecer las estrategias de conservación (Thompson et al. 2001). El presente estudio da a conocer la riqueza y presencia de las especies del género Thamnophis en distintas provincias biogeográficas, áreas decretadas para la conservación y zonas con diferente tipo de impacto antropogénico.

El análisis de la riqueza de especies mostró que en las provincias del Eje Neovolcánico Transversal y de Oaxaca se presentó la mayor riqueza específica para el género Thamnophis, tal como se ha encontrado en otros grupos taxonómicos (Escalante et al., 2002; Ochoa-Ochoa y Flores-Villela, 2006). Adicionalmente, se encontró una alta riqueza específica en la Sierra Madre Oriental y Occidental. Las especies que componen al clado mexicano (de Queiroz, et al., 2002) aportaron la mayor riqueza a las provincias del Eje Neovolcánico Transversal y Oaxaca; mientras que las especies del clado de amplia distribución aportaron la mayor riqueza a las provincias de la Sierra Madre Oriental y Occidental, las cuales también se distribuyen en Norteaméricay Centroamérica (Rossman et al., 1996).

La riqueza de las especies de culebras del género Thamnophis presentó 2 patrones espaciales, uno altitudinal y otro climático. En el patrón altitudinal, la mayor riqueza específica se encontró en las provincias biogeográficas y RTP con altitudes mayores de $1000 \mathrm{~m}$ snm. En este sentido, las especies endémicas de México (44\%) se encontraron a altitudes mayores de $1000 \mathrm{~m}$ y presentaron distribuciones geográficas menores que las especies que se distribuyen tanto en Norteamérica como en Centroamérica (Rossman et al., 1996; de Queiroz et al., 2002). La elevada riqueza específica a tales altitudes pudo deberse a la especiación alopátrica de esas especies al final del Pleistoceno (McCormack et al., 2008). En este periodo existió un incremento en la temperatura ambiental que redujo los ambientes templados a mayores altitudes, lo cual promovió el aislamiento geográfico de sus poblaciones y posteriormente su especiación (de Queiroz et al., 2002). El anterior patrón de especiaciòn también se ha registrado para aves (Baker, 2008; McCormack et al.; 2008), murciélagos (Dolan, 1982), peces (Mateos et al., 2002) y plantas (Graham, 1989) de los sistemas montañosos de México.

El patrón climático de riqueza específica se caracterizó por una mayor riqueza de especies en climas templados a muy fríos y una menor riqueza en los semicálidos a cálido subhúmedos y de semiáridos a muy áridos. Una posible explicación ecológica a este patrón es la asociación de las especies de Thamnophis a cuerpos de agua para termorregularse y para obtener sus presas (véase revisión en Rossman et al., 1996). Esta asociación se hace más evidente en las especies que habitan las provincias con climas de semicalidos a muy áridos, como son la Sonorense y las del Altiplano del Sur y del Norte, donde la disponibilidad de agua limita la presencia de más especies de este género. Por ejemplo, Thamnophis hammondii, que fue la única especie en la provincia de Baja California, se restringe a relictos de ambientes menos secos, los cuales fueron resultado del proceso de desertificación ocurrido en la península al final del Pleistoceno (Grismer, 1994). El género presentó bajas riquezas específicas en las provincias y RTP con climas de semicálidos a cálido subhúmedos, como es el caso de las provincias biogeográficas de Yucatán, el Petén, el Soconusco y del Golfo de California. En el caso de la provincia del Golfo de México, se pueden encontrar hasta 6 especies en las áreas con clima templado, mientras que en las de clima cálido potencialmente pueden encontrarse de 1 a 2 especies. Thamnophis proximus y $T$. marcianus, especies con distribuciones fragmentadas en climas cálidos del sureste de México, son representativas de los límites más sureños, tanto para estas especies como para el género (Rossman et al., 1996).

El patrón climático de riqueza específica también tiene una explicación filogenética. Evidencias moleculares demuestran que las especies basales del clado mexicano y del clado de amplia distribución se encuentran en climas templados del Eje Neovolcanico Transversal y la Sierra Madre Occidental, mientras que la especie más derivada (Thamnophis fulvus) se distribuye al sur del istmo de Tehuantepéc (de Queiroz et al., 2002), tanto en climas templados como cálidos. Además, el $76.8 \%$ de las RTP ocupadas por las especies del clado mexicano fueron del grupo oriental y occidental-central, donde domina el clima templado. Por lo que posiblemente un linaje ancestral de estas especies de Thamnophis se originó en zonas templadas y posteriormente radio hacia climas relativamente más cálidos donde se presentan las especies derivadas. Esta posibilidad puede explorarse mediante un estudio de la estructura filogeográfica de las especies que ayude a explicar los patrones de flujo génico interespecífico (Rossman et al., 1996).

La ordenación de las RTP, en función de la presenciaausencia de las especies del género Thamnophis, presentó una tendencia espacial bien definida en las especies que lo componen. El grupo de RTP californiano mostró especies del oeste, de las grandes planicies de los Estados Unidos (de Queiroz et al., 2002), que son representativas de los límites más sureños de la distribución de las especies de este grupo (Rossman et al., 1996). Los grupos norteño y atlántico sólo contuvieron especies de amplia distribución, 
las cuales también están en los otros 3 grupos. Las RTP de los grupos oriental, pacífico y occidental-central abarcaron las 10 especies endémicas del clado mexicano, las 4 del grupo godmani y 2 endémicas del clado de amplia distribución (de Queiroz et al. 2002).

Las RTP se crearon con el objetivo de preservar la mayor variedad de condiciones ambientales con posibilidades reales para la conservación de una amplia diversidad de especies (Arriaga et al., 2000). El resultado del análisis de complementariedad mostró que las RTP contienen todas las especies de culebras del género Thamnophis. En este sentido, más del 30\% de la distribución geográfica del $56.7 \%$ de las especies endémicas del género Thamnophis coincidió con las RTP y sólo la distribución geográfica de 2 especies endémicas presentó una coincidencia de menos del 10\% con las RTP. Por lo que el propósito de las RTP de conservar la mayor diversidad de especies se cumple para las especies de culebras del género Thamnophis.

Las áreas con la mayor complementariedad son congruentes con otros estudios que las analizaron en las Áreas Naturales Protegidas (Santos-Barrera et al., 2004), los reptiles en celdas de $0.5^{\circ}$ (Ochoa-Ochoa y Flores-Villela, 2006) y en aves (Álvarez-Mondragón y Morrone, 2004). La RTP con el complemento residual más bajo, es decir, con el mayor número de especies de reptiles complementarias, fue la de las Sierras del norte de Oaxaca y Mixte, que también lo fue para las especies de aves (ÁlvarezMondragón y Morrone, 2004).

La mayor riqueza de especies del género Thamnophis se encontró en el Eje Neovolcánico Transversal; sin embargo, en esta área las especies están sujetas a diversos factores antropogénicos que pueden reducir el tamaño de sus poblaciones o su distribución geográfica, entre los que se encuentran: 1) la mayor densidad poblacional en sus RTP; 2) del 0 al 70\% de vegetación nativa; 3) del $30-70 \%$ de superficie agrícola; 4) el sobre-pastoreo; 5) la baja disponibilidad de agua, y 6) su localización dentro de las regiones hidrológicas con mayor número de habitantes (SEMARNAT, 2006). En similares circunstancias se encuentran la provincia de Oaxaca, donde se perdió del 3 al $10 \%$ de la cobertura vegetal entre los años de 1993 y 2002. En contraste, las provincias de la Sierra Madre Oriental y Occidental, que aportan el mayor porcentaje a la distribución del género, presentan menor presión antropogénica debido a que mantienen entre el 60 y $100 \%$ de la cobertura de vegetación nativa, el porcentaje de pérdida de vegetación nativa entre los años de 1993 y 2003 es bajo (0-3.5\%) y las densidades poblacionales en sus RTP son menores a 2 habitantes por celda. Por lo tanto, las áreas prioritarias para conservación de estas especies, y considerando la actividad antropogénica, son las que se encuentran en la provincia del Eje Neovolcánico Transversal.
Al considerar el porcentaje de la distribución de las especies dentro de las RTP y el porcentaje de coincidencia con áreas modificadas por el hombre, se encontró que las especies con mayor presión antropogénica fueron Thamnophis scalaris, T. scaliger, T. melanogaster y T. postremus; las 4 son endémicas de México y sólo de T. melanogaster se cuenta con estudios ecológicos (Rossman et al., 1996). Por lo que, dada la presión antropogénica y lo restringido de sus distribuciones, es prioritario realizar estudios que señalen los factores locales y regionales que determinan la distribución y abundancia de estas especies. También es necesario realizar estudios de las especies endémicas con distribuciones geográficas restringidas, como es el caso de T. bogerti, T. conanti, T. exsul, T. godmani, T. lineri, T. mendax o T. sumichrasti, de las que sólo se cuenta con la descripción taxonómica y con datos biológicos aislados (Rossman et al. 1996). La prioridad de realizar estudios en estas especies se fundamenta en que las especies con pequeñas distribuciones geográficas y que habitan en condiciones restringidas tienden a presentar mayores riesgos de extinción (Purvis 2000).

Los resultados obtenidos en el presente estudio fueron derivados de la distribución potencial de las especies del género Thamnophis. Sin embargo, la distribución de las especies probablemente sea menor a lo que registran Rossman et al. (1996), debido a que tiende a ser espacialmente discontinua (Pulliam, 2000) y gran parte de su hábitat está modificado por la actividad antropogénica (SEMARNAT, 2006). En especies de aves y mamíferos del noroeste de México es evidente que la distribución potencial es menor que la existente (Ortega-Huerta y Peterson, 2004). Sin embargo, también es evidente en el anterior estudio que hay una gran congruencia entre una y otra distribución, difiriendo sólo en la extensión ocupada por cada una. Si el anterior patrón se presenta en las especies del género Thamnophis, entonces, los resultados aquí presentados pueden reflejar la tendencia geográfica en la riqueza específica de las especies del género Thamnophis.

Considerando que más del $30 \%$ de la distribución de 11 especies de culebras se da en áreas modificadas por la actividad antropogénica, se sugiere realizar estudios que relacionen la variación fenotípica y genotípica ante gradientes ambientales, ya sea naturales o modificados por el hombre, con el objetivo de identificar los factores ambientales y antropogénicos que están relacionados con la presencia de estas especies (Pulliam, 2000); asimismo, que se elaboren mapas de calidad de hábitat para predecir la distribución potencial de las especies e identificar los factores ambientales relacionados con su distribución regional (Phillips et al. 2007), lo cual se puede realizar aun con las especies con pocos registros de colecta. Además, se debe considerar que potencialmente existen más especies 
que las descritas actualmente. Por ejemplo, se ha planteado que $T$. cyrtopsis o $T$. eques presentan linajes crípticos (de Queiroz et al., 2002; Conant, 2003), como fue el caso del complejo godmani (Rossman y Burbrink, 2005).

Este estudio es una primera aproximación en el análisis de la distribución de la riqueza específica del género Thamnophis y plantea una hipótesis de donde potencialmente existe la mayor riqueza específica, la que deberá probarse en subsecuentes estudios de campo.

\section{Agradecimientos}

La realización del proyecto fue el resultado de la estancia posdoctoral de CSVB, apoyada por el CONACYT. Agradecemos a un revisor anónimo por los valiosos y acertados comentarios que incrementaron la calidad del presente manuscrito. CSVB agradece a Karina Domínguez, a Corina y a Selene Venegas por su apoyo moral durante la realización del proyecto.

\section{Literatura citada}

Álvarez-Mondragón, E y J. J. Morrone. 2004. Propuestas de áreas para la conservación ed aves en México, empleando herramientas panbiogeográficas e índices de complementariedad. Interciencia 29:112-120.

Arriaga, L., J. M. Espinoza, C. Aguilar, E. Martínez, L. Gómez y E. Loa. 2000. Regiones terrestres prioritarias de México. Comisión Nacional para el Conocimiento y uso de la Biodiversidad, México, D. F. 609 p.

Badgley, C. y D. L. Fox. 2000. Ecological biogeography of North American mammals: species density and ecological structure in relation to environmental gradients. Journal of Biogeography 27:1437-1467.

Baker, A. J. 2008. Islands in the sky: the impact of Pleistocene climate cycles on biodiversity. Journal of Biology 7:1-4.

Bestelmeyer, B., J. R. Miller y J. A. Wiens. 2003. Applying species diversity theory to land management. Ecological Applications 13:1750-1761.

CONABIO (Comisión Nacional para el Conocimiento y Uso de la Biodiversidad). 1998. La diversidad biológica de México: un estudio de país. México, D.F. 341 p.

Conant, R. 2003. Observations on garter snakes of the Thamnophis eques complex in the Lakes of Mexico's Transvolcanic Belt, with descriptions of new taxa. American Museum Novitates 3406:1-64.

de Queiroz, A., R. Lawson y J. A. Lemos-Espinal. 2002. Phylogenetic relationships of North American Garter Snakes (Thamnophis) based on four mitochondrial genes: How much DNA sequence is enough? Molecular Phylogenetics and Evolution 22:315-329.

Dolan, P. G. 1982. Systematics of Middle American Mastiff bats (Molossus). Tesis, doctorado en Filosofía, Texas Tech University, Austin. 151 p.

Escalante, T., D. Espinosa y J. J. Morrone. 2002. Patrones de distribución geográfica de los mamíferos terrestres de México. Acta Zoológica Mexicana (n.s.) 87:47-65.

Flores-Villela, O. y L. Canseco-Márquez. 2004. Nuevas especies y cambios taxonómicos para la herpetofauna de México. Acta Zoológica Mexicana 20:115-144.

Graham, A. 1989. Late tertiary paleoaltitudes and vegetational zonation in Mexico and Central America. Acta Botanica Neerlandica 38:471-424.

Grismer, L. L. 1994. The origin and evolution of the Peninsular herpetofauna of Baja California, México. Herpetological Natural History 2:51-106.

Humphries, C. J., R. I. Van Wright, y P. H. Williams. 1991. Biodiversity reserves: setting new priorities for the conservation of wildlife. Parks 2:34-38.

Jackson, D. A., K. M. Somers y H. H. Harvey. 1989. Similarity coefficients: measures of co-occurrence and associations or simply measures of occurrence? The American Naturalist 133:436-453.

Jongman, R. H. G., C. J. F. Ter Braak y O. F. R. Van Tongeren. 1995. Data analysis in community and landscape ecology. Cambridge University Press, Cambrige. 299 p.

Mateos, M., O. I. Sanjur y R. C. Vrijenhoek. 2002. Historical biogeography of the livebearing fish genus Poeciliopsis (Poeciliidae: Cyprinodontiformes). Evolution 56:972-984.

McCormack, J. E., T. A. Peterson, E. Bonaccorso y T. B. Smith. 2008. Speciation in the highlands of Mexico: genetic and phenotypic divergence in the Mexican jay (Aphelocoma ultramarina). Molecular Ecology 17: 2505-2521

Morrone, J. J. 2005. Hacia una síntesis biogeográfica de México. Revista Mexicana de Biodiversidad 76:207-252.

Ochoa-Ochoa, L. M. y O.A. Flores-Villela. 2006. Áreas de diversidad y endemismo de la herpetofauna mexicana. Universidad Nacional Autónoma de México/ Comisión Naconal para el Conocimiento y Uso de la Biodiversidad, México, D. F. 211 p.

Ortega-Huerta, M. A. y A. T. Peterson. 2004. Modelling spatial patterns of biodiversity for conservation prioritization in North-eastern Mexico. Diversity and Distribution 10:39-54.

Phillips, S. J., R. P. Anderson, y R. E. Schapire. 2006. Maximum entropy modeling of species geographic distributions. Ecological Modelling 190:231-259.

Peterson, T. A., M. A. Ortega-Huerta, J. Bartley, V. SánchezCordero, J. Soberón, R. H. Buddenmeier y D. Stockwell. 2002. Future projections for Mexican faunas under global climate change scenarios. Nature 416:626-628.

Pulliam, H. R. 2000. On the relationship between niche and distribution. Ecology Letters 3:349-361.

Rey, B. J. M. y E. de la Montaña. 2003. Identifying areas of highvalue vertebrate diversity for strengthening conservation. 
Biological Conservation 114:357-370.

Rossman, D. A. y F. T. Burbrink. 2005. Species limits within the Mexican garter snakes of the Thamnophis godmani complex. Occasional Paper of the Museum of Natural Science 1-44.

Rossman, D. A., N. B. Ford y R. A. Seigel. 1996. The garter snakes: evolution and ecology. University of Oklahoma Press, Norman, $331 \mathrm{p}$.

Santos-Barrera, G., J. Pacheco y G. Ceballos. 2004. Áreas prioritarias para la conservación de los reptiles y anfibios de México. Biodiversitas 57:1-6.

SEMARNAT (Secretaría de Medio Ambiente y Recursos Naturales). 2006. El medio ambiente en México 2005: en resumen. México, D.F., 91 p.

Thompson, J. N., O. J. Reichman, P. J. Morin, G. A. Polis, M. E. Power, R. W. Sterner, C. A. Couch, L. Gough, R. Holt, D. U. Hooper, F. Keesing, C. R. Lovell, B. T. Milne, M. C. Molles, D. W. Roberts y S. Y. Strauss. 2001. Frontier of ecology. Bioscience 51:15-24.

Torres, R. J. M. 2004. Estudio de tendencias y perspectivas del sector forestal en América Latina al año 2020: informe nacional. In FAO-SEMARNAT. 2002. Estado de la Información Forestal en México, Información para el desarrollo forestal sostenible, Monografía de países. Volumen 11, Santiago de Chile, 279 p. 
